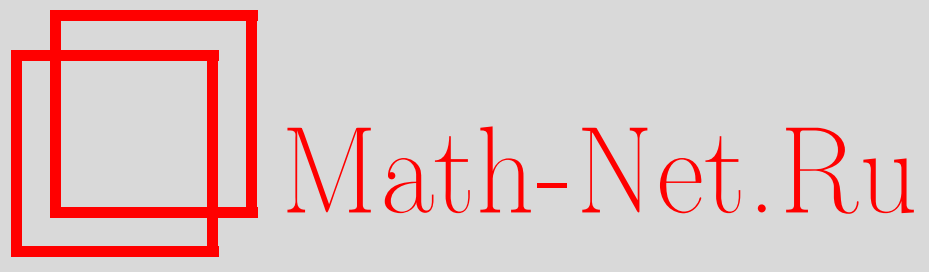

А. О. Ремизов, Особенности в трехмерных аффинноуправляемых системах со скалярным управлением, УМH, 2007, том 62, выпуск 4, 175-176

DOI: https://doi.org/10.4213/rm7008

Использование Общероссийского математического портала Math-Net.Ru подразумевает, что вы прочитали и согласны с пользовательским соглашением http://www . mathnet.ru/rus/agreement

Параметры загрузки:

IP: 34.229 .108 .108

26 апреля 2023 г., 16:01:16

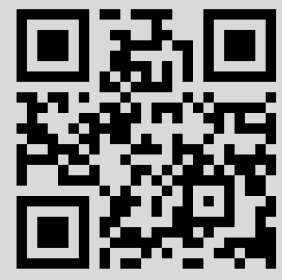




\section{Особенности в трехмерных аффинно-управляемых системах со скалярным управлением}

\section{А. О. Ремизов}

В геометрической теории управления встречаются системы, в которых вторая производная гамильтониана $H$ по управлению $u$ вдоль экстремали тождественно равна нулю. В частности, это происходит в аффинных по управлению системах, когда максимум (или минимум) $H$ по $u \in U$ достигается не на границе, а внутри множества $U$. Такие экстремали называются вполне особыми. Здесь мы рассмотрим поле вполне особых экстремалей типичной управляемой системы вида

$$
\frac{d x}{d t}=f_{0}(x)+u f_{1}(x), \quad x \in X(\operatorname{dim} X=3), \quad u \in U \subset \mathbb{R}^{1},
$$

где $f_{i}: X \rightarrow T X-$ гладкие (т. е. класса $C^{\infty}$ ) векторные поля на гладком трехмерном многообразии $X$, множество допустимых управлений состоит из кусочно гладких локально ограниченных функций $u(t)$ со значениями в $U$. Необходимые условия оптимальности первого и второго порядка для таких систем получены в [1]. Гамильтониан $H$ равен $h_{0}+u h_{1}$, где $h_{i}=\left\langle f_{i}(x), p\right\rangle, p \in T_{x}^{*} X$, треугольные скобки означают действие ковектора на вектор. Введем обозначения $h_{i_{1}, \ldots, i_{k}}=\left\{h_{i_{1}}, h_{i_{2}, \ldots, i_{k}}\right\}$ и $f_{i_{1}, \ldots, i_{k}}=\left[f_{i_{1}}, f_{i_{2}, \ldots, i_{k}}\right]$, где фигурные (квадратные) скобки означают скобки Пуассона (Ли) функций (векторных полей). Гамильтонова система принципа максимума Понтрягина для (1) имеет вид

$$
\frac{d x}{d t}=f_{0}(x)+u f_{1}(x), \quad \frac{d p}{d t}=-\frac{\partial h_{0}}{\partial x}-u \frac{\partial h_{1}}{\partial x} .
$$

Вполне особые экстремали удовлетворяют равенству $h_{1}=0$, из которого посредством двукратного дифференцирования в силу (2) получаем $h_{01}=0$ и $h_{001}-u h_{110}=0$. Не содержащие $u$ уравнения $h_{1}=0$ и $h_{01}=0$ определяют многообразие $S \subset T^{*} X$, содержащее все вполне особые экстремали, а уравнение $h_{001}-u h_{110}=0$ позволяет выразить управление $u$ через переменные $(x, p) \in T^{*} X$. Подставляя $u=\frac{h_{001}}{h_{110}}$ в $(2)$, мы получаем на $S$ векторное поле $V$, не содержащее $u$ и определенное во всех точках многообразия $S$, в которых $h_{110} \neq 0$. Фазовые кривые этого поля суть вполне особые экстремали, а их проекции на пространство $X$ - соответствующие экстремальные траектории (1). При этом возникают особенности двух типов.

Особенности первого типа - это точки, в которых $h_{110}=h_{001}=0$ (случай $h_{110}=0$, $h_{001} \neq 0$ исключается из рассмотрения, так как $u$ принимает бесконечное значение). Для исследования вполне особых экстремалей (фазовых кривых поля $V$ ) в окрестности таких точек рассмотрим поле, полученное умножением $V$ на $h_{110}$ :

$$
\dot{x}=h_{110} f_{0}(x)+h_{001} f_{1}(x), \quad \dot{p}=-h_{110} \frac{\partial h_{0}}{\partial x}-h_{001} \frac{\partial h_{1}}{\partial x},
$$

где точка сверху означает дифференцирование по новой переменной $\tau$, связанной с временем $t$ равенством $\dot{t}=h_{110}$. Предположим, что поля $f_{0}, f_{1}$ и $f_{1}, f_{01}$ линейно независимы. Тогда $S$ является гладким 4-мерным многообразием и проектирование $\pi: S \rightarrow X$ регулярно (субмерсия коранга 1 ). Введя на $S$ локальные координаты $(x, \omega)$, где $p=\omega f_{1} \times f_{01}(x)$, представим его в виде $S=S_{+} \cup S_{-}$, где компоненты связности $S_{ \pm}$ определяются знаком $\omega$. Проектирование $\pi: S \rightarrow X$ переводит поле (3), умноженное на $|\omega|^{-1}$, с компонент $S_{ \pm}$в векторные поля

$$
\dot{x}= \pm\left(e_{1} f_{0}+e_{0} f_{1}\right),
$$

Работа выполнена при поддержке гранта SFRH/BPD/26138/2005 (Португалия). 
где $e_{1}=\left\langle f_{1} \times f_{01}, f_{110}\right\rangle, e_{0}=\left\langle f_{1} \times f_{01}, f_{001}\right\rangle$. В случае общего положения множество особых точек полей (4) - регулярная кривая $e_{0}=e_{1}=0$, трансверсальная $\operatorname{span}\left(f_{0}, f_{1}\right)$; спектр линеаризации поля (4) в любой особой точке равен $(\lambda,-\lambda, 0)$, где $\lambda \neq 0$ вещественное или чисто мнимое число, и имеет место стандартное разложение касательного пространства:

$$
T_{x} X=T_{x}^{\mathrm{c}} \oplus T_{x}^{\mathrm{s}} \oplus T_{x}^{\mathrm{u}}(\operatorname{Re} \lambda \neq 0) \quad \text { или } \quad T_{x} X=T_{x}^{0} \oplus T_{x}^{ \pm \lambda}(\operatorname{Re} \lambda=0),
$$

$T_{x}^{\mathrm{s}}$ и $T_{x}^{\mathrm{u}}$ для полей $(4)$ с противоположными знаками меняются местами. Предположим, что поверхность $K: e_{1}=0$ трансверсальна $T_{x}^{\mathrm{s}}$ и $T_{x}^{\mathrm{u}}$ в вещественном и $T_{x}^{ \pm \lambda}$ в мнимом случае. Тогда в мнимом случае фазовые кривые полей (4) - спирали, пересекающие криминанту и, следовательно, не отвечающие никакому допустимому управлению. Согласно [2], в вещественном случае ростки полей (4) с квадратичной частью общего положения конечно-гладко (с любой наперед заданной степенью гладкости) орбитально эквивалентны росткам полей

$$
\pm\left(\xi \frac{\partial}{\partial \xi}-\eta \frac{\partial}{\partial \eta}+\xi \eta \frac{\partial}{\partial \zeta}\right)
$$

с первым интегралом $\xi \eta$. В новых координатах проходящие через особые точки фазовые кривые (траектории системы (1)) - это прямые, параллельные оси $\xi$ или $\eta$. Переключение управления в особой точке происходит в соответствии с условием второго порядка $h_{110} \leqslant 0$, см. [1].

Особенности второго типа связаны с нерегулярностью проектирования $\pi: S \rightarrow X$. Образы таких точек определяются условием $f_{1} \times f_{01}=0$ и образуют кривую $\psi \subset X$, на которой обращаются в нуль функции $e_{0}$ и $e_{1}$. В случае общего положения среди компонент поля $f_{1} \times f_{01}$ две имеют независимые дифференциалы, кривая $\psi$ регулярна и трансверсальна $\operatorname{span}\left(f_{0}, f_{1}\right)$. Тогда для каждой точки $x \in \psi$ ее прообраз $\pi^{-1}(x)$ двумерен и $S_{\psi}=\pi^{-1}(\psi)$ - гладкое трехмерное подмногообразие $S$, определяющее стратификацию $S=S_{\psi} \cup S_{+} \cup S_{-}$, где $S_{ \pm}$определены, как и раньше, знаком $\omega$ в $p=\omega f_{1} \times f_{01}(x)$. Поле (3) трансверсально $S_{\psi}$ и его проекция на $X$ не касается кривой $\psi$, поэтому все вполне особые экстремали содержатся в стратах $S_{ \pm}$, за исключением лишь самой точки пересечения экстремали с $S_{\psi}$. Проекция поля $(3)$ с $S_{ \pm}$ на $X$ дает поля (4), определенные на множестве $\pi^{-1}\left(S_{+} \cup S_{-}\right)=X \backslash \psi$. Продолжая по непрерывности поля (4) на все пространство $X$, получаем, что кривая $\psi$ становится центральным многообразием $W^{\mathrm{c}}$ этих полей, состоящим из их особых точек. Спектр линеаризации этих полей в любой особой точке равен $\pm(\lambda, \lambda, 0)$, где $\lambda \neq 0$ - вещественное число, причем жорданова форма линейной части диагональна. Согласно [2], ростки этих полей конечно-гладко (с любой наперед заданной степенью гладкости) орбитально эквивалентны росткам полей

$$
\pm\left(\xi \frac{\partial}{\partial \xi}+\eta \frac{\partial}{\partial \eta}+0 \frac{\partial}{\partial \zeta}\right)
$$

\section{Список литературы}

[1] А.А. Аграчев, Ю.Л. Сачков, Геометрическая теория управления, Физматлит, М., 2004. [2] А. О. Ремизов, "Многомерная конструкция Пуанкаре и особенности поднятых полей для неявных дифференциальных уравнений”, СМФН, 19 (2006), 131-170.

А. О. Ремизов (А. O. Remizov)

Universidade do Porto, Portugal

E-mail: remizov@caravan.ru
Представлено М. И. Зеликиным Принято редколлегией 24.05.2007 